\title{
Highly Efficient Ru-Pseudohalide Catalysts for Olefin Metathesis
}

Jay C. Conrad, Henrietta H. Parnas, Jennifer L. Snelgrove and Deryn E. Fogg*

Center for Catalysis Research and Innovation, Department of Chemistry, University of Ottawa, 10 Marie Curie, Ottawa ON, Canada, K1N 6N5

\section{SUPPORTING INFORMATION}

\section{Contents:}

1. Experimental procedures.

2. ${ }^{1} \mathrm{H}$ and ${ }^{13} \mathrm{C}\left\{{ }^{1} \mathrm{H}\right\}$ NMR spectra for $\mathbf{8 a}$ and $\mathbf{8 b}$.

3. References.

*Corresponding author. E-mail: dfogg@science.uottawa.ca. Fax: (613) 562-5170 


\section{Experimental Procedures.}

General Procedures. Synthetic procedures were carried out at room temperature $\left(22^{\circ} \mathrm{C}\right)$ under $\mathrm{N}_{2}$ or argon, using standard Schlenk or drybox techniques. Dry, oxygen-free solvents were obtained using an Anhydrous Engineering solvent purification system, and stored over Linde $4 \AA$ molecular sieves. $\mathrm{CDCl}_{3}$ was distilled from $\mathrm{CaH}_{2}$, then degassed by consecutive freeze/pump/thaw cycles and stored over activated sieves. $\mathrm{C}_{6} \mathrm{D}_{6}$ was dried over activated sieves (Linde $4 \AA$ ) and degassed by consecutive freeze/pump/thaw cycles. ${ }^{1} \mathrm{H}$ NMR (300 or $500 \mathrm{MHz}$ ), ${ }^{31} \mathrm{P}$ NMR (121 or $\left.202 \mathrm{MHz}\right)$ and ${ }^{13} \mathrm{C}(75$ or $125 \mathrm{MHz})$ spectra were recorded on a Bruker Avance-300 or Bruker Avance-500 spectrometer. IR spectra were measured on a Bomem MB100 IR spectrometer. Microanalyses and ICP-AES (inductively coupled plasma atomic emission spectroscopy) analyses for $\mathrm{Ru}$ content were carried out by Guelph Chemical Laboratories Ltd., Guelph, Ontario. Complexes $\mathbf{1},{ }^{1} \mathbf{2 a},{ }^{2}$ and $\mathbf{7},{ }^{3}$ and substrates $\mathbf{1 7},{ }^{4} \mathbf{1 9},{ }^{4} \mathbf{2 3},{ }^{5}$ and $\mathbf{2 5}^{5}$ were prepared by literature procedures. Substrates 9, 11, 13 and $\mathbf{2 1}$ (Aldrich) were distilled and degassed before use; 15 (Lancaster) was degassed only. Phenols and TlOEt were purchased from Aldrich and used without purification.

Note: The toxicity of thallium (particularly in the +1 oxidation state) is well established, and the subject has been recently reviewed. ${ }^{6}$ Care must be taken to prevent introduction into the body by inhalation, by ingestion via contaminated hands or gloves, or through the skin. All thallium reagents and wastes, including contaminated solvents, were handled using double-glove and secondary containment procedures, with separate disposal of all wastes in accordance with government regulations. 
General Procedure for Formation of $\mathbf{T l O C}_{6} \mathbf{X}_{5}$ salts. To an oven-dried flask containing a solution of $\mathrm{HOC}_{6} \mathrm{X}_{5}(5.0 \mathrm{~g})$ in $100 \mathrm{~mL}$ dry toluene was added 1.0 equiv TlOEt. The solution was stirred for $8 \mathrm{~h}$, after which the solvent was removed under vacuum. The resulting white solid was washed with hexanes $(3 \times 8 \mathrm{~mL})$, then dried under vacuum to yield a fine white powder, which was handled under nitrogen in a drybox. Yield 80-90\%.

$\mathbf{R u C l}_{2}(\mathbf{C H P h})(\mathrm{IMes})(\mathbf{p y})_{2}$ (3a). The complex was prepared by the procedure reported for the $\mathrm{H}_{2}$ IMes analogue. ${ }^{7}$ Addition of a minimum volume of pyridine (ca. $2 \mathrm{~mL}$ ) to solid $\mathrm{RuCl}_{2}(\mathrm{CHPh})(\mathrm{IMes})\left(\mathrm{PCy}_{3}\right)(\mathbf{2 a})(872 \mathrm{mg}, 1.03 \mathrm{mmol})$ caused a color change from red to green. After 10 minutes, $20 \mathrm{~mL}$ of pentane was added, and the suspension was chilled to $-35{ }^{\circ} \mathrm{C}$. The resulting green solid was filtered off and dried under vacuum. Yield $610 \mathrm{mg}(82 \%) .{ }^{1} \mathrm{H}$ NMR $\left(\mathrm{C}_{6} \mathrm{D}_{6}, \delta\right) 20.02(\mathrm{~s}, 1 \mathrm{H}, \mathrm{RuCH}), 9.33\left(\mathrm{~d},{ }^{2} J_{\mathrm{HH}}=4.3,2 \mathrm{H}, \mathrm{py}\right), 8.55\left(\mathrm{~d},{ }^{2} J_{\mathrm{HH}}=4.7,2 \mathrm{H}, \mathrm{py}\right), 8.25(\mathrm{~d}$, $\left.{ }^{2} J_{\mathrm{HH}}=7.5,2 \mathrm{H}, \mathrm{Ph}\right), 7.21-7.14\left(\mathrm{~m}, 1 \mathrm{H}, \mathrm{Ph}\right.$, overlaps with solvent peak), $6.94\left(\mathrm{t},{ }^{2} J_{\mathrm{HH}}=7.6,2 \mathrm{H}\right.$, $\mathrm{Ph}), 6.78\left(\mathrm{t},{ }^{2} J_{\mathrm{HH}}=7.2,1 \mathrm{H}, \mathrm{py}\right), 6.50(\mathrm{~s}, 4 \mathrm{H}, \mathrm{Mes} \mathrm{Ar}, \mathrm{m}-\mathrm{CH}), 6.43\left(\mathrm{t},{ }^{2} J_{\mathrm{HH}}=5.9,2 \mathrm{H}, \mathrm{py}\right), 6.30-$ $6.27(\mathrm{~m}, 3 \mathrm{H}, \mathrm{py}, \mathrm{NCH}), 6.00\left(\mathrm{t},{ }^{2} J_{\mathrm{HH}}=6.2,2 \mathrm{H}, \mathrm{py}\right), 2.50\left(\mathrm{~s}, 12 \mathrm{H}\right.$, Mes $\left.\mathrm{CH}_{3}\right), 1.99(\mathrm{~s}, 6 \mathrm{H}, \mathrm{Mes}$ $\left.\mathrm{CH}_{3}\right) .{ }^{13} \mathrm{C}\left\{{ }^{1} \mathrm{H}\right\} \mathrm{C}_{6} \mathrm{D}_{6} \delta 317.8$ (s, RuCH), 184.8 (s, NCN), 153.8 (s, $\left.\operatorname{Ar} C\right), 152.5$ (s, $\operatorname{Ar} C H$ ), 150.9 (s, $\operatorname{Ar} C \mathrm{H}$ ), 138.4 (s, $\operatorname{Ar} C H$ ), 138.3 (s, $\operatorname{Ar} C H$ ), 137.7 (s, $\operatorname{Ar} C$ ), 135.2 (s, $\operatorname{Ar} C$ ), 134.1 (s, $\operatorname{Ar} C$ ), 131.1 (s, $\operatorname{Ar} C H$ ), 129.2 (s, $\operatorname{Ar} C H$ ), 129.1 (s, $\operatorname{Ar} C H$ ), 128.3 (s, $\operatorname{Ar} C H$ ), 127.9 (s, $\operatorname{Ar} C H$ ), 124.9 (s, $\operatorname{Ar} C H$ ), 122.8 (s, $\operatorname{Ar} C H$ ), 122.6 (s, $\mathrm{Ar} C H$ ), 21.1 (s, $\left.\mathrm{CH}_{3}\right), 19.5$ (s, $\mathrm{CH}_{3}$ ). IR (Nujol, $\left.\mathrm{cm}^{-1}\right)$ : v (C=C) 1593 (w). Anal. Calcd. $\mathrm{C}_{38} \mathrm{H}_{40} \mathrm{~N}_{4} \mathrm{Ru}$ : C, 62.98; H, 5.56; N, 7.73\%. Found C, 63.21; H, $5.94 ; \mathrm{N}, 7.61 \%$. 
$\mathbf{R u C l}\left(\mathbf{O C}_{6} \mathbf{C l}_{5}\right)(\mathbf{C H P h})(\mathrm{IMes})(\mathbf{p y})(\mathbf{8 a})$. To a solution of 3a $(200 \mathrm{mg}, 0.276 \mathrm{mmol})$ in $20 \mathrm{~mL}$ benzene was added solid $\mathrm{TlOC}_{6} \mathrm{Cl}_{5}(130 \mathrm{mg}, 0.276 \mathrm{mmol})$. The reaction was stirred for $8 \mathrm{~h}$, then filtered through Celite and stripped to dryness. The green residue was redissolved in toluene (5 $\mathrm{mL}$ ) and filtered through neutral alumina. The filtrate was concentrated to $\sim 0.5 \mathrm{~mL}$, after which $40 \mathrm{~mL}$ of hexanes was added over 5 minutes. The mixture was cooled to $-35{ }^{\circ} \mathrm{C}$. A green precipitate deposited over a period of 5 hours. This was filtered off and dried under high vacuum. Yield $170 \mathrm{mg}(70 \%)$. Yields are limited by high solubility. ${ }^{1} \mathrm{H}$ NMR $\left(\mathrm{C}_{6} \mathrm{D}_{6}, \delta\right) 19.78(\mathrm{~s}, \mathrm{RuCH}$, 1H), 8.26 (br, 2H, py or $\mathrm{Ph}), 8.03$ (br, 2H, Ph or py), $7.15-7.11$ (m, 1H, py or Ph, overlaps with solvent peak), $6.88\left(\mathrm{t}, J_{\mathrm{HH}}=9,2 \mathrm{H}\right.$, py or $\left.\mathrm{Ph}\right), 6.74(\mathrm{~s}, 2 \mathrm{H}, \mathrm{Mes} \mathrm{Ar}, \mathrm{m}-\mathrm{CH}), 6.33(\mathrm{~s}, 2 \mathrm{H}, \mathrm{Mes} \mathrm{Ar}$, $\mathrm{m}-\mathrm{CH}), 6.18\left(\mathrm{t}, J_{\mathrm{HH}}=7.2,1 \mathrm{H}\right.$, py or Ph), $6.15(\mathrm{~s}, 2 \mathrm{H}, \mathrm{NCH}), 5.90\left(\mathrm{br} \mathrm{t},{ }^{2} J_{\mathrm{HH}}=6.3,2 \mathrm{H}\right.$, py or Ph), 2.46 (s, 6H, Mes $\mathrm{CH}_{3}$ ), 2.07 (s, 6H, Mes $\left.\mathrm{CH}_{3}\right), 2.00\left(\mathrm{~s}, 6 \mathrm{H}\right.$, Mes $\left.\mathrm{CH}_{3}\right) \cdot{ }^{13} \mathrm{C}\left\{{ }^{1} \mathrm{H}\right\} \mathrm{C}_{6} \mathrm{D}_{6} \delta 314.9(\mathrm{~s}$, $\mathrm{RuCH}), 181.4(\mathrm{~s}, \mathrm{NCN}), 161.5$ (s, $\operatorname{Ar} C), 152.7$ (s, $\operatorname{Ar} C$ ), 151.5 (s, $\operatorname{Ar} C H$ ), 138.9 (s, $\operatorname{Ar} C$ ), 137.1 (s, $\operatorname{Ar} C$ ), 137.0 (s, $\operatorname{Ar} C$ ), 135.7 (s, $\operatorname{Ar} C H$ ), 129.9 (s, $\operatorname{Ar} C H$ ), 129.2 (s, $\operatorname{Ar} C H$ ), 129.1 (s, $\operatorname{Ar} C H$ ), 129.0 (s, $\operatorname{Ar} C H$ ), 127.9 (s, $\operatorname{Ar} C H$ ), 127.8 (s, $\operatorname{Ar} C H$ ), 124.3 (s, $\operatorname{Ar} C H$ ), 122.9 (s, NCHCHN), 20.9 (s, $\left.\mathrm{CH}_{3}\right), 18.6$ (s, $\left.\mathrm{CH}_{3}\right), 17.9$ (s, $\mathrm{CH}_{3}$ ). Anal. Calcd. $\mathrm{C}_{39} \mathrm{H}_{35} \mathrm{Cl}_{6} \mathrm{~N}_{3} \mathrm{ORu}$ : C, 53.50; H, 4.03; N, 4.80\%. Found C, 53.94; H, 3.99; N, 4.81\%.

$\operatorname{RuCl}\left(\mathbf{O C}_{6} \mathbf{B r}_{5}\right)(\mathbf{C H P h})(\mathbf{I M e s})(\mathbf{p y})(\mathbf{8 b})$. To a solution of 3a $(200 \mathrm{mg}, 0.276 \mathrm{mmol})$ in $20 \mathrm{~mL}$ benzene was added $\mathrm{TlOC}_{6} \mathrm{Br}_{5}(191 \mathrm{mg}, 0.276 \mathrm{mmol})$. The reaction was stirred overnight, then filtered through Celite and stripped of solvent. The green powder was redissolved in $0.5 \mathrm{~mL}$ toluene, then $20 \mathrm{~mL}$ hexanes was added over $5 \mathrm{~min}$. The mixture was cooled to $-35^{\circ} \mathrm{C}$ for several hours, over which time a green precipitate deposited. This was filtered off and dried under vacuum. Yield $257 \mathrm{mg}(85 \%)$. ${ }^{1} \mathrm{H}$ NMR $\left(\mathrm{C}_{6} \mathrm{D}_{6}, \delta\right) 19.68(\mathrm{~s}, \mathrm{Ru}=\mathrm{CH}, 1 \mathrm{H}), 8.07$ (br, 4H, py 
$+\mathrm{Ph}), 7.16-7.11\left(\mathrm{~m}, 1 \mathrm{H}\right.$, py or $\mathrm{Ph}$, overlaps with solvent peak), $6.87\left(\mathrm{t}, J_{\mathrm{HH}}=7.5,2 \mathrm{H}\right.$, py or Ph), 6.75 (s, 2H, Mes Ar, m-CH), 6.36 (s, 2H, Mes Ar, m-CH), $6.12-6.13(\mathrm{~m}, 3 \mathrm{H}, \mathrm{NCH}$, py or Ph), $5.96-5.84\left(\mathrm{~m}, 2 \mathrm{H}\right.$, py or Ph), $2.46\left(\mathrm{~s}, 6 \mathrm{H}, \mathrm{Mes}_{\mathrm{CH}}\right), 2.10\left(\mathrm{~s}, 6 \mathrm{H}, \mathrm{Mes} \mathrm{CH}_{3}\right), 1.98(\mathrm{~s}, 6 \mathrm{H}$, Mes $\left.\mathrm{CH}_{3}\right) .{ }^{13} \mathrm{C}\left\{{ }^{1} \mathrm{H}\right\}\left(\mathrm{CDCl}_{3}\right) \delta 315.6(\mathrm{~s}, \mathrm{Ru}=\mathrm{CH}), 181.5(\mathrm{~s}, \mathrm{NCN}), 163.2(\mathrm{~s}, \mathrm{Ar} C), 152.5(\mathrm{~s}, \mathrm{Ar}$ C), 151.3 (s, $\operatorname{Ar} C H$ ), 139.3 (s, $\operatorname{Ar} C$ ), 137.5 (s, $\operatorname{Ar} C$ ), 137.1 (s, $\operatorname{Ar} C$ ), 136.1 (s, $\operatorname{Ar} C H$ ), 133.7 (s, $\operatorname{Ar} C$ ), 129.9 (s, $\operatorname{Ar} C H$ ), 129.7 (s, $\operatorname{Ar} C H$ ), 129.4 (s, $\operatorname{Ar} C H$ ), 129.1 (s, $\operatorname{Ar} C H$ ), 128.2 (s, $\operatorname{Ar}$ C), 127.8 (s, $\operatorname{Ar} C \mathrm{H}), 125.5$ (s, $\operatorname{Ar} C$ ), 124.8 (s, $\operatorname{Ar} C H$ ), 123.3 (s, NCHCHN), 120.1 (s, $\operatorname{Ar} C$ ), 116.4 (s, $\operatorname{Ar} C), 107.8$ (s, $\operatorname{Ar} C$ ), $21.3\left(\mathrm{~s}, C \mathrm{H}_{3}\right), 18.8\left(\mathrm{~s}, C \mathrm{H}_{3}\right), 17.9\left(\mathrm{~s}, C \mathrm{H}_{3}\right)$. Anal. calcd $\mathrm{C}_{39} \mathrm{H}_{35} \mathrm{Br}_{5} \mathrm{ClN}_{3} \mathrm{ORu}: \mathrm{C}, 42.67 ; \mathrm{H}, 3.21 ; \mathrm{N}, 3.83 \%$. Found C, 42.77; H, 3.12; N, 3.81\%.

\section{Representative Procedures for Ring-Closing Metathesis Reactions.}

(a) RCM to form five-membered rings, using 0.05 mol\% catalyst. To a small reaction vessel containing $1.0 \mathrm{~mL} \mathrm{CDCl}{ }_{3}$ was added 10.0 microliters $(0.05 \mu \mathrm{mol})$ of a stock solution of 7 in $\mathrm{CHCl}_{3}(5.0 \mathrm{mg}$ in $1.062 \mathrm{~mL} ; 5.00 \mathrm{mM})$. Diethyldiallyl malonate $9(24 \mathrm{mg}, 0.10 \mathrm{mmol})$ was then added (final $[\mathrm{S}]=0.10 \mathrm{M},[\mathrm{C}]=0.05 \mathrm{mM}$ ). The rapidly-stirred reaction mixture was heated under $\mathrm{N}_{2}$ in an oil bath set to $65^{\circ} \mathrm{C}$, and conversions were monitored by removing samples for GC analysis, using tetrahydronaphthalene as internal standard; $\pm 3 \%$ in replicate runs. Complete conversion to product was confirmed by NMR analysis.

(b) RCM to form five-membered rings, using $0.5 \mathrm{~mol} \%$ catalyst. To a small reaction vessel containing $2.08 \mathrm{~mL} \mathrm{CDCl}_{3}$ was added 229 microliters $(1.04 \mu \mathrm{mol})$ of a stock solution of $\mathbf{8 b}$ in $\mathrm{CHCl}_{3}(5.0 \mathrm{mg}$ in $1.0 \mathrm{~mL} ; 4.55 \mathrm{mM})$. Diethyldiallyl malonate $9(50 \mathrm{mg}, 0.208 \mathrm{mmol})$ was then added; final $[\mathrm{S}]=0.1 \mathrm{M},[\mathrm{C}]=0.5 \mathrm{mM}$. The rapidly-stirred reaction mixture was heated under $\mathrm{N}_{2}$ in an oil bath set to $65^{\circ} \mathrm{C}$, and conversions were monitored by removing samples for GC 
analysis, using tetrahydronaphthalene as internal standard; $\pm 3 \%$ in replicate runs. Complete conversion to product was confirmed by NMR analysis.

(c) RCM to form macrocycles 17 and 19. A three-neck round-bottom flask was fitted with a condenser and two addition funnels. Solutions of catalyst $\left(6.4 \times 10^{-6} \mathrm{~mol}\right.$ in $10 \mathrm{~mL}$ solvent $)$ and substrate $\left(12.8 \times 10^{-5} \mathrm{~mol}\right.$ in $10 \mathrm{~mL}$ solvent $)$ were independently added dropwise, over 15 minutes, to $10 \mathrm{~mL}$ of refluxing solvent under argon $\left(17: \mathrm{CH}_{2} \mathrm{Cl}_{2} ; 19: \mathrm{C}_{6} \mathrm{H}_{6}\right)$. Aliquots were removed periodically for $\mathrm{GC}$ analysis, beginning at 15 minutes. GC retention times and integrations were confirmed with samples of authentic material, for which response factors were independently measured.

Chromatographic Purification. Following RCM of diethyldiallyl malonate (9) using 5 mol \% $\mathrm{Ru}$, as described above, the solvent was stripped off to yield a dark oil, which was purified by flash chromatography on silica gel. Product $\mathbf{1 0}$ was isolated in $98 \%$ yield by elution with $15 \mathrm{~mL}$ of EtOAc-hexanes, and the Ru content was measured by inductively-coupled plasma atomic emission spectroscopy (ICP-AES). Using 15\% EtOAc:hexanes: 1, 2450 ppm; 7, 600 ppm. Using 5\% EtOAc-hexanes: 7, 8a, 8b, <100 ppm; 1, 30,900 ppm, 2a, 57,900 ppm. 
Part 2. NMR Spectra for New Pseudohalide Complexes.

(a) ${ }^{1} \mathrm{H}$ NMR Spectrum of $\operatorname{RuCl}\left(\mathrm{OC}_{6} \mathrm{Cl}_{5}\right)(\mathrm{CHPh})(\mathrm{IMes})(\mathrm{py}), 8 \mathrm{a}\left(\mathrm{C}_{6} \mathrm{D}_{6}, 300 \mathrm{MHz}\right)$

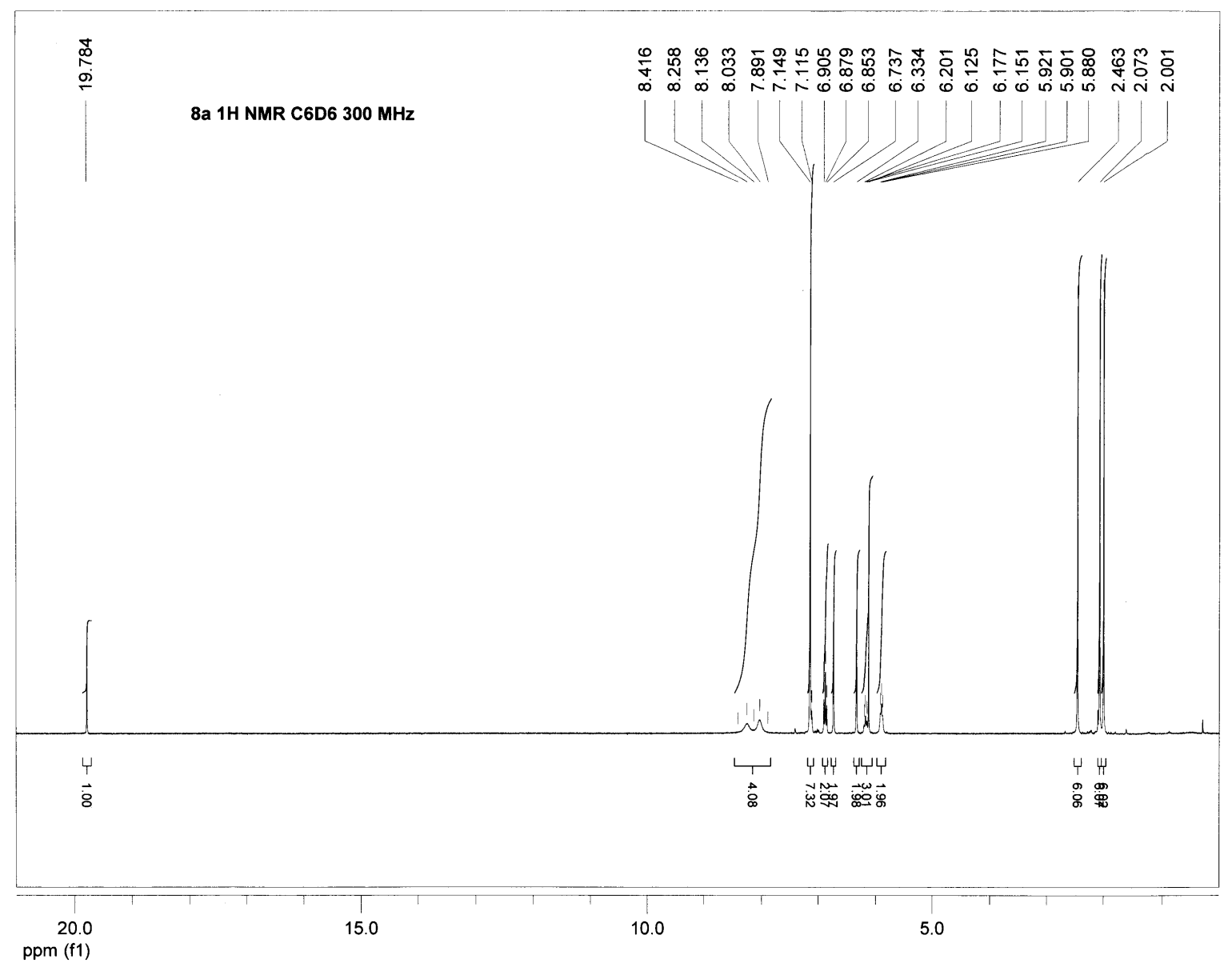


Part 2. NMR Spectra for New Pseudohalide Complexes (cont'd).

(b) ${ }^{13} \mathrm{C}\left\{{ }^{1} \mathrm{H}\right\}$ NMR Spectrum of $\mathrm{RuCl}\left(\mathrm{OC}_{6} \mathrm{Cl}_{5}\right)(\mathrm{CHPh})(\mathrm{IMes})(\mathrm{py}), 8 \mathrm{a}\left(\mathrm{C}_{6} \mathrm{D}_{6}, 75 \mathrm{MHz}\right)$

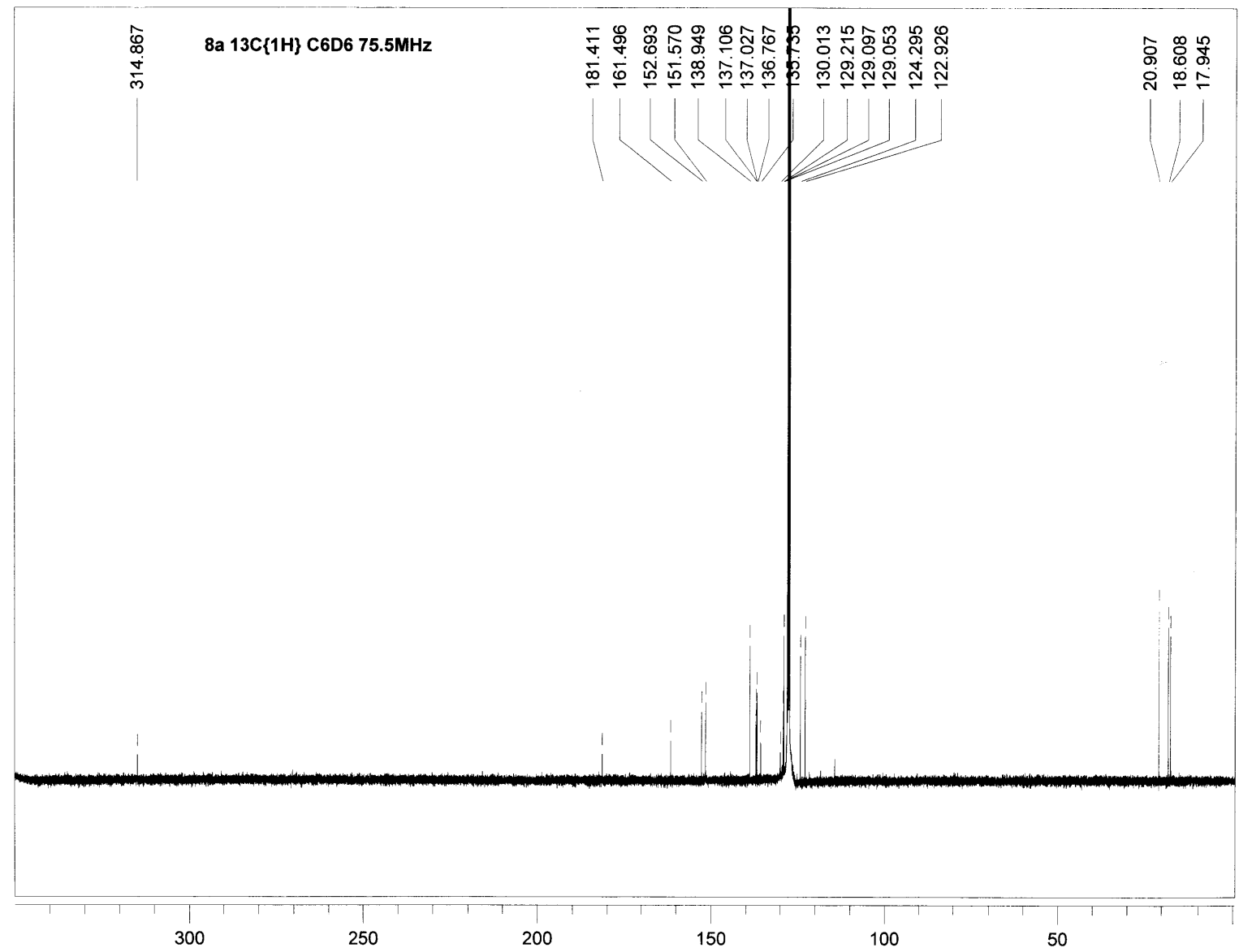


Part 2. NMR Spectra for New Pseudohalide Complexes (cont'd).

(c) ${ }^{1} \mathrm{H}$ NMR Spectrum of $\mathrm{RuCl}\left(\mathrm{OC}_{6} \mathrm{Br}_{5}\right)(\mathrm{CHPh})(\mathrm{IMes})(\mathrm{py}), 8 \mathrm{~b}\left(\mathrm{C}_{6} \mathrm{D}_{6}, 300 \mathrm{MHz}\right)$

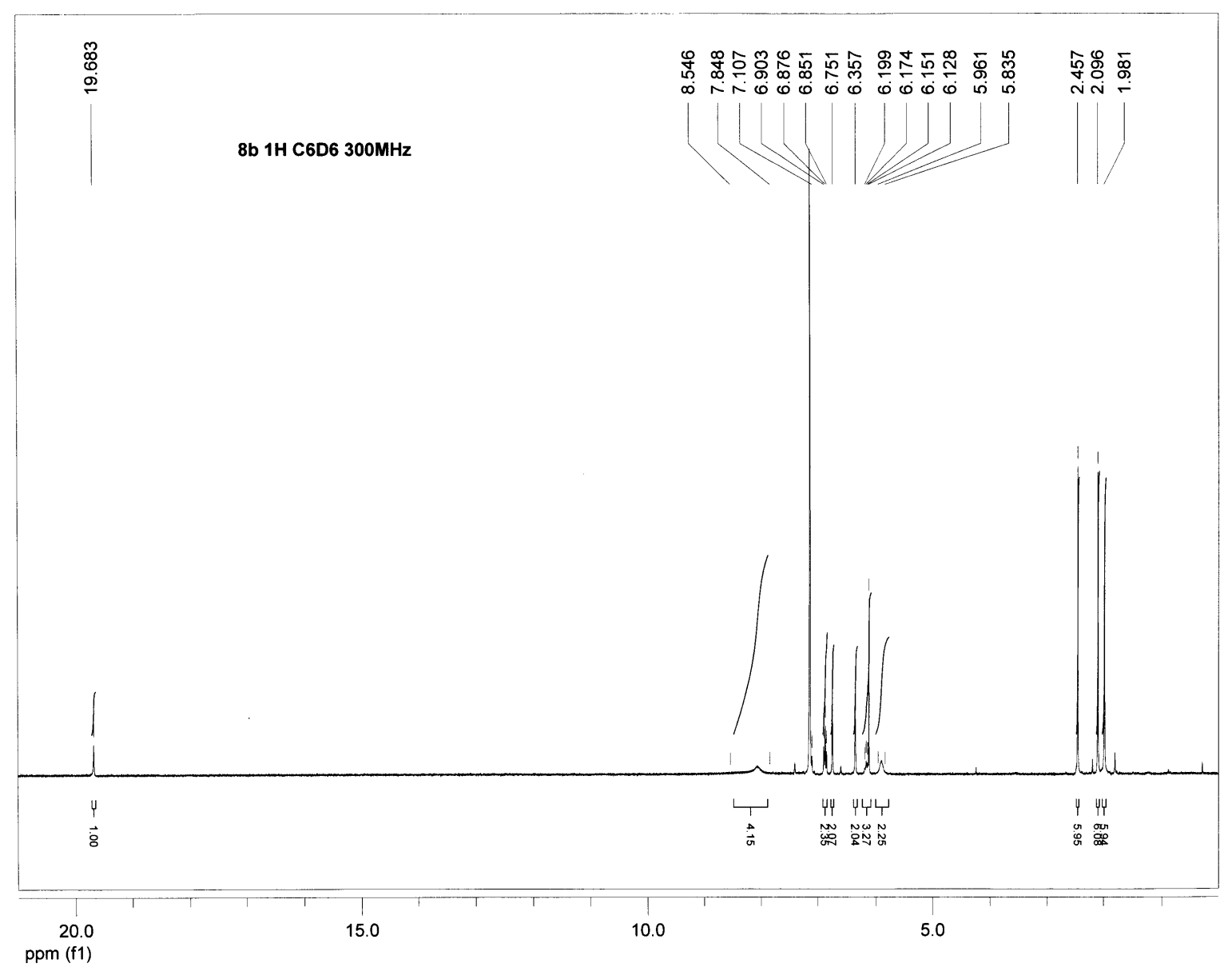


Part 2. NMR Spectra for New Pseudohalide Complexes (cont'd).

(d) ${ }^{13} \mathrm{C}\left\{{ }^{1} \mathrm{H}\right\}$ NMR spectrum of $\operatorname{RuCl}\left(\mathrm{OC}_{6} \mathrm{Br}_{5}\right)(\mathrm{CHPh})(\mathrm{IMes})(\mathrm{py}), 8 \mathrm{~b}\left(\mathrm{C}_{6} \mathrm{D}_{6}, 75 \mathrm{MHz}\right)$
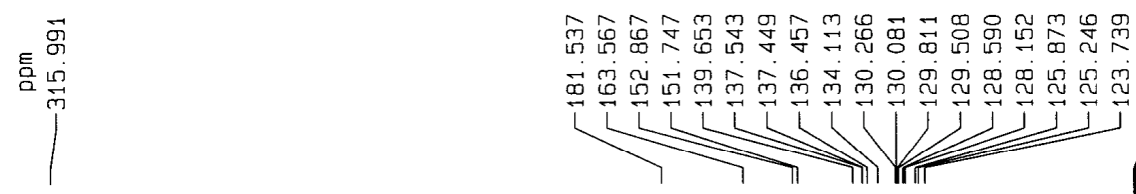

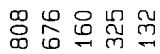

तi $\dot{\sim}$ 유
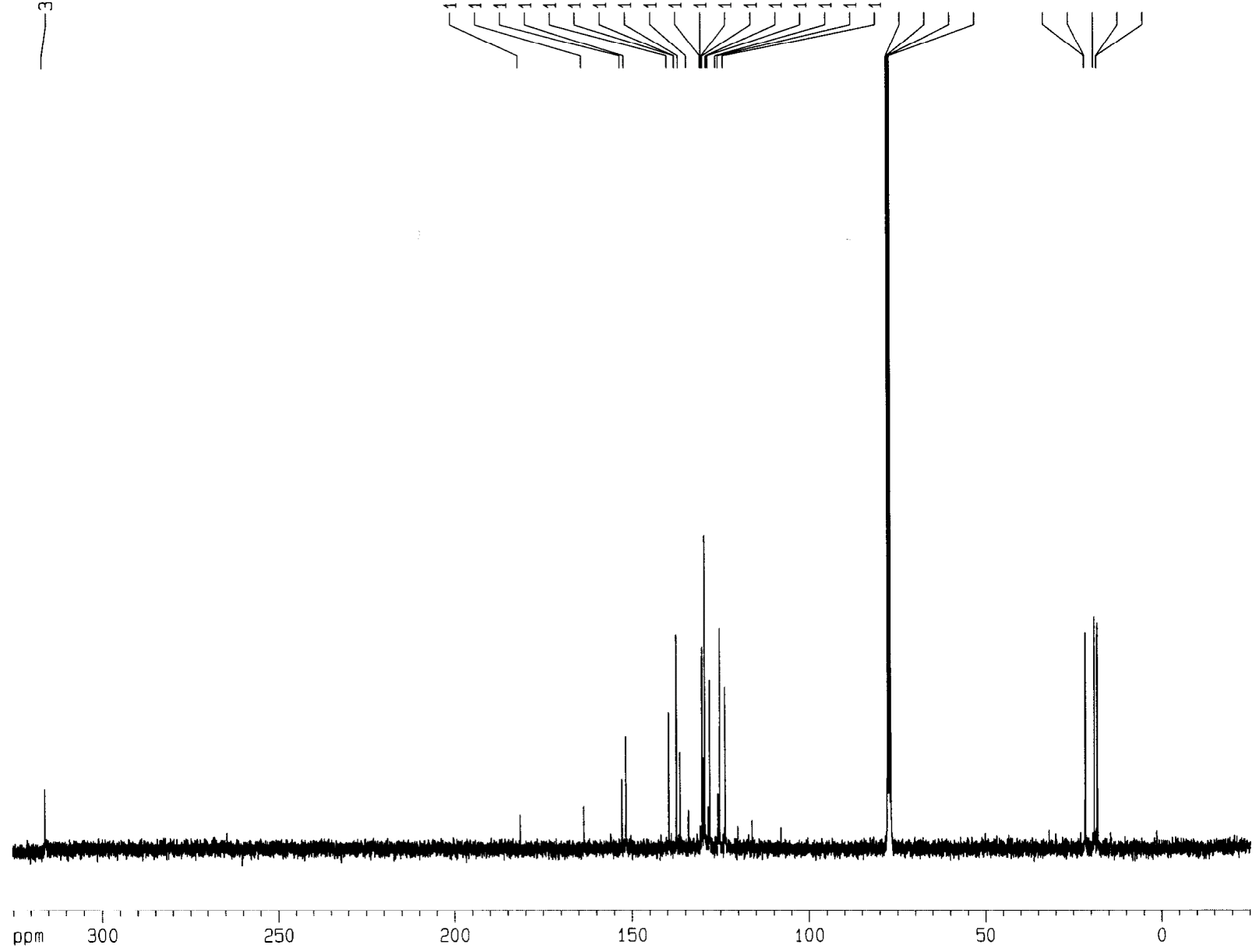


\section{References.}

(1) Schwab, P.; Grubbs, R. H.; Ziller, J. W. J. Am. Chem. Soc. 1996, 118, 100-10.

(2) Huang, J.; Stevens, E. D.; Nolan, S. P.; Peterson, J. L. J. Am. Chem. Soc. 1999, 121, 26742678.

(3) Conrad, J. C.; Amoroso, D.; Czechura, P.; Yap, G. P. A.; Fogg, D. E. Organometallics 2003, $22,3634-3636$.

(4) Fürstner, A.; Langemann, K. Synthesis 1997, 792-803.

(5) Piquet, M.; Bruneau, C.; Dixneuf, P. H. Chem. Commun. 1998, 2249-2250.

(6) Galván-Arzate, S.; Santamaría, A. Toxicol. Lett. 1998, 99, 1.

(7) Sanford, M. S.; Love, J. A.; Grubbs, R. H. Organometallics 2001, 20, 5314-5318. 